\title{
SAXES, a high resolution spectrometer for resonant x-ray emission in the 400-1600 eV energy range
}

\author{
G. Ghiringhelli, A. Piazzalunga, C. Dallera, G. Trezzi, and L. Braicovich \\ Dipartimento di Fisica, Politecnico di Milano, Piazza Leonardo da Vinci 32, I-20133 Milano, Italy \\ T. Schmitt, V. N. Strocov, R. Betemps, and L. Patthey \\ Swiss Light Source, Paul Scherrer Institut, CH-5232 Villigen PSI, Switzerland \\ X. Wang and M. Grioni \\ Institut de Physique des Nanostructures, Ecole Polytechnique Fédéderale de Lausanne, \\ CH-1015 Lausanne, Switzerland
}

(Received 26 July 2006; accepted 2 October 2006; published online 14 November 2006)

\begin{abstract}
We present a $5 \mathrm{~m}$ long spectrometer for soft $\mathrm{x}$ rays to be used at a synchrotron radiation beamline for resonant $\mathrm{x}$-ray emission spectroscopy and resonant inelastic $\mathrm{x}$-ray scattering in the $400-1600 \mathrm{eV}$ energy range. It is based on a variable line spacing spherical grating (average groove density of $3200 \mathrm{~mm}^{-1}, R=58.55 \mathrm{~m}$ ) and a charge coupled device two dimensional detector. With an x-ray spot on the sample of $10 \mu \mathrm{m}$, the targeted resolving power is higher than 10000 at all energies below $1100 \mathrm{eV}$ and better than 7000 at $1500 \mathrm{eV}$. The off-line tests made with $\mathrm{Al}$ and $\mathrm{Mg} K \alpha_{1,2}$ fluorescence emissions indicate that the spectrometer can actually work at 12000 and 17000 resolving power at the $L_{3}$ edges of $\mathrm{Cu}(930 \mathrm{eV})$ and of $\mathrm{Ti}(470 \mathrm{eV})$, respectively. SAXES (superadvanced $\mathrm{x}$-ray emission spectrometer) is mounted on a rotating platform allowing to vary the scattering angle from $25^{\circ}$ to $130^{\circ}$. The spectrometer will be operational at the ADRESS (advanced resonant spectroscopies) beamline of the Swiss Light Source from 2007. () 2006 American Institute
\end{abstract} of Physics. [DOI: 10.1063/1.2372731]

\section{INTRODUCTION}

The availability of third generation synchrotron radiation sources and of even better sources in the coming years (diffraction limited storage rings and free electron lasers) is opening the access to unprecedented energy resolution in inelastic x-ray scattering spectroscopy. The progress of x-ray sources has to be accompanied by consistent advancements of the instrumentation, in order to maximize the future impact of these techniques onto physics, chemistry, and materials sciences. In this context the soft $\mathrm{x}$-ray range (below $\sim 1700 \mathrm{eV}$ photon energy) is of particular importance for resonant spectroscopies due to the presence of absorption edges of silicon, carbon, nitrogen, oxygen, $3 d$ transition metals ( $3 d \mathrm{TMs}$ ), and lanthanides. Moreover $\mathrm{x}$-ray emission spectroscopy (XES) and resonant inelastic x-ray scattering (RIXS) can benefit from the particularly strong resonant enhancements at the $\mathrm{O} K$ edge, $3 d \mathrm{TM} L_{2,3}$ edges, and rare earth $M_{4,5}$ edges in the $400-1600 \mathrm{eV}$ range. ${ }^{1-3}$ These experiments require a synchrotron radiation beamline delivering a highly monochromatic x-ray beam onto a small area of the sample and a spectrometer capable of measuring the emission spectrum, at fixed incident photon energy, usually with a parallel acquisition over a convenient energy window. Considerable efforts have to be devoted to the improvement of spectrometer performances in terms of energy resolving power and detection efficiency. In fact, at these energies the scattering cross sections are always very small and the signal is further reduced by the necessity of using grazing incidence gratings in the spectrometers, which drastically reduce the instrument angular acceptance. After the pioneering works by Callcott et $a l^{4}{ }^{4}$ and by Nordgren et al..$^{5}$ whose instrument, also commercially available, has been widely used in these years, other groups have developed spectrometers following a similar layout. ${ }^{6-11}$ The effort for getting narrower spectral linewidth (LW) has been pursued both in the very soft $\mathrm{x}$-ray range (typically up to $100 \mathrm{eV}$ covering the $M$ edges of the $3 d \mathrm{TMs}$ ) and at higher energies (e.g., the $L$ edges of $3 d \mathrm{TMs}$ and the $M_{4,5}$ resonances of lanthanides from about 450 to $1600 \mathrm{eV}$ ) where the goal is more difficult: a ten times higher resolving power is required to get the same linewidth. On the other hand $L$ edges usually have stronger resonances and better defined intermediate states in the scattering process, and interesting perspectives are given by the scattering angle dependence: the photon momentum is not negligible and the transferred momentum versus transferred energy behavior can be studied.

In this field an important project is based at the Swiss Light Source (SLS) where a new beamline called ADRESS (advanced resonant spectroscopies) has been designed to be dedicated particularly to RIXS and to high energy angular resolved photoemission in the $400-1600 \mathrm{eV}$ energy range. In RIXS the goals of achieving very high energy resolution and of exploring scattering angle dependence have required to jointly design the beamline and the spectrometer. In this article we present SAXES (superadvanced $\mathrm{x}$-ray emission spectrometer), the high resolution instrument that is being installed at the ADRESS beamline. SAXES has already been tested off line with conventional x-ray sources. We present 
here its novelties with respect to previous instruments. The instrument is an evolution of AXES (advanced x-ray emission spectrometer) ${ }^{7,12}$ built by some of us and working since 1995 at the beamline ID08 of the ESRF in Grenoble. ${ }^{13}$ Our goal was now to reach 12000 resolving power at the $L_{3}$ edge of $\mathrm{Cu}(930 \mathrm{eV})$, meaning $\sim 80 \mathrm{meV} \mathrm{LW}$, in consideration of the great importance of high $T_{c}$ superconducting cuprates. More in general we targeted a resolving power higher than 10000 over the whole energy range. With a beamline monochromator having roughly the same resolving power or slightly better, this would give a combined LW around $100 \mathrm{meV}$ full width at half maximum (FWHM), i.e., considerably better than any published RIXS measurements at the $\mathrm{Cu} L_{3}$ edge. $^{14,15}$ As shown below the spectrometer has reached this goal. On the other side the beamline monochromator has been designed to reach 30000 resolving power over the whole energy range.

One important experimental parameter in XES and RIXS is the signal intensity that has often limited the actual working resolution which had to be reduced in order to get sufficient statistical quality in the spectra. In our case when passing from AXES at the ESRF to SAXES at the SLS, we should obtain comparable intensities on the detector. In fact, the optical layout is very similar and a factor of about 3 should be lost because of the reduction of the horizontal angular acceptance due to the longer distance of the detector from the sample $(5 \mathrm{~m}$ instead of $2.2 \mathrm{~m})$. Another loss should come from the grating efficiency (higher groove density in SAXES than in AXES), partially compensated by a better quality of the groove profile in the new grating. On the other hand a gain should come from a much better optical matching between the beamline and the spectrometer and, at least at low energies, from higher intensity delivered by the ADRESS beamline with respect to ID08. These estimates make us confident about the actual feasibility of the high resolution RIXS spectra using SAXES at the ADRESS beamline.

\section{THE OPTICS OF SAXES}

\section{A. Concepts on the layout}

Up to now the spectrometers for XES and RIXS in the soft X-ray range, where Bragg crystals cannot be employed, have been designed according to two schemes. Some systems are based on one single optical element, a grazing incidence concave diffraction grating dispersing and focusing the radiation onto a position sensitive detector to be positioned in the appropriate place depending on the energy of the spectrum. Other systems combine a concave mirror with a plane grating in order to separate the dispersion and focusing actions. The latter should provide greater angular acceptance (typically a factor of 2-3), but it is obviously more complicated to build and to operate.

In our project we opted for the single optical element scheme. This choice was done not only to privilege simplicity but also to have a system where the minimization of all the higher order optical aberrations is easily obtained over the whole energy range. When using variable line spacing (VLS) gratings with fixed radius of curvature and fixed po-
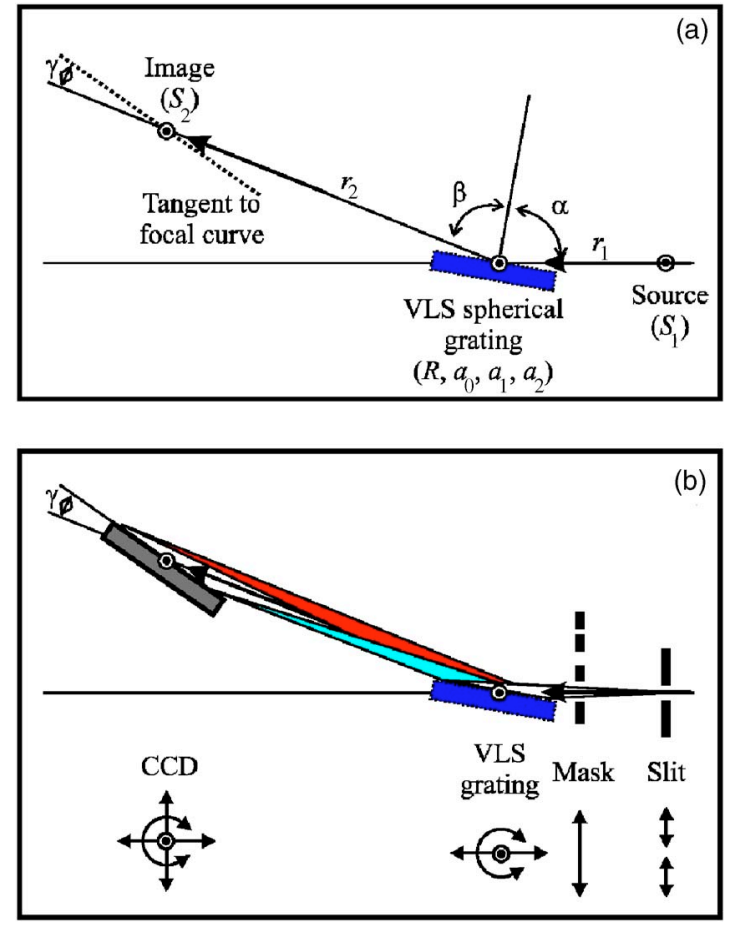

FIG. 1. (Color online) The schematic layout of the SAXES spectrometer, which is based on a VLS spherical grating and a two dimensional position sensitive CCD detector. Panel (A): the main optical parameter symbols are defined. Panel (B): the main mechanical components are sketched together with their degrees of freedom. The entrance slit width can be regulated with a $1 \mu \mathrm{m}$ accuracy. The grating can be moved along the entrance arm and can be rotated to change $r_{1}$ and $\alpha$, respectively. The CCD detector can be displaced to follow the focusing curve and can be rotated to change the incidence angle $\gamma$. A mask with a set of apertures of different vertical widths is placed just before the grating to allow a regulation of the illuminated footprint on the grating, in order to have a full control of optical aberrations.

sition, it is not possible to cancel the coma aberration at more than one energy. That is why in SAXES the grating can be repositioned (in terms of distance from the source and incidence angle) for minimizing the LW broadening due to the high order optical aberrations. The same procedure is possible also in the two optical element systems, but it seems to us more difficult to implement.

The layout of SAXES is shown in Fig. 1 (bischematic side view). Panel (A) shows the parameters to be considered in the optical design (entrance and exit arm lengths $r_{1}$ and $r_{2}$, incidence and diffraction angles $\alpha$ and $\beta$, source size $S_{1}$, detector effective spatial resolution $S_{2}$, angle of glancing incidence on the focal curve around a given energy $\gamma$, and VLS grating parameters $R, a_{0}, a_{1}$, and $a_{2}$ ). In panel (B) we show the main available degrees of freedom provided by our mechanics: the entrance slit width, the longitudinal grating position, the grating pitch angle, the vertical and horizontal detector positions, and its pitch angle. In addition a set of apertures in a vertically movable mask can be used to change the length of the grating illuminated area (in order to further control optical aberrations). For any photon energy the grating has to be put at the right position and angle; the first internal order diffracted beam can be collected by the detector located in the corresponding focal position and oriented to be tangential to the focal curve. Owing to the use of a two dimensional detector, a whole spectrum can then be recorded 
in parallel, i.e., nothing needs to be scanned during the spectrum acquisition. The spectrometer is installed on a rotating girder platform, which provides additional degrees of freedom (roll, yaw, as well as transverse and vertical movements) essential for the alignment of the spectrometer to the sample and incident x-ray beam.

\section{B. Choice of the parameters}

The choice of the optical parameters is dictated by both optical and mechanical boundary conditions. As explained above the grating position and orientation have to be adjustable to allow the minimization of the optical aberrations. The minimum source size $S_{1}$ is given either by the width of the entrance slit or by the height of the illuminated spot on the sample: a good refocusing after the exit slit of the beamline is vital in order to maximize the detection efficiency even when using a very narrow entrance slit. Moreover, if the spot height is sufficiently small it will be possible, at least in some occasions, to use SAXES without input slit in order to get the maximum efficiency. A spot size down to $9 \mu \mathrm{m}$ (FWHM) should be feasible at the ADRESS beamline due to an ellipsoidal mirror refocusing with a theoretical demagnification of about 5.5. In the design we have assumed $S_{1}$ $=5-10 \mu \mathrm{m}$. If in the future smaller sizes will be attained, this will increase mostly the luminosity and only to some extent the resolution which will be in that case limited by the spatial resolution of the detector.

The spatial resolution of the charge couples device (CCD) detector determines the spectrometer virtual exit slit. Modern CCD detectors for soft $\mathrm{x}$ rays are back illuminated and thinned to reduce the surface dead layer; their detection efficiency is probably above $80 \%$ in our energy range. They are very user friendly and their effective spatial resolution is around $25 \mu \mathrm{m}$, irrespective of the pixel size, as a consequence of split events, as shown below. The only way to gain in spatial resolution is thus to mount the CCD at grazing incidence: in our case we chose $\gamma=20^{\circ}$ (see Fig. 1 that is not to scale) so that the effective exit slit width is $S_{2}=8-9 \mu \mathrm{m}$, of the same order as $S_{1}$. This means that, in the optimal layout, the magnification by the grating is around unity at the first order.

The optimal use of the CCD mounted at grazing incidence implies that the spectrum is dispersed on a focal locus well approximated by the surface of the CCD. The control of this parameter (the angle $\gamma$ ) is obtained through the appropriate choice of the VLS parameters as proposed by Osborn and Callcott in Ref. 16. One has also to fix the total length of the spectrometer: a longer instrument can reach higher resolving power but the horizontal angular acceptance, determined by the size of the detector, is then reduced. For SAXES we established a maximum length of around $5 \mathrm{~m}$, i.e., more than two times the length of AXES at the ESRF; this is also the maximum space available for the end station at the ADRESS beamline. The loss in angular acceptance should be counterbalanced by a better optical matching between beamline and spectrometer, as well as by a larger photon flux of the ADRESS beamline. Another important consideration concerns the average ruling density and the VLS parameters. The present manufacturing technology allowed us to safely reach $a_{0}=3200 \mathrm{~mm}^{-1}$ and a maximum variation of the local density along the grating of $10 \%$.

The actual optical design went first through a search for the first approximation parameters using the analytical expressions for VLS gratings, ${ }^{16-18}$ and then checked and optimized with ray tracing using the SHADOW code. ${ }^{19}$ The two methods give coincident results except for the cancellation of coma and other higher order aberrations. Moreover, some discrepancies in the equations on the coma aberrations can be found between Refs. 16, 18, and 20. It is not our purpose to solve here those discrepancies (see also the Appendix). The ultimate values are those given by the ray tracing. The logical procedure that we used is the following.

First we assign the $E_{0}$ central energy $\left(E_{0}=900 \mathrm{eV}\right)$ where the instrument is optimized. For $E_{0}$ we assign the total length $L$, the included angle $\theta_{0}=\left(\alpha_{0}+\beta_{0}\right) / 2$, the incidence angle on the detector $\gamma$, the average ruling density $a_{0}$, the reference source size $S_{1}$, the effective spatial resolution of the detector $S_{2}$, and the diffraction order $k$ (in our case first internal order, $k=+1)$. We define the local ruling density as $a(w)=a_{0}+a_{1} w+a_{2} w^{2}+\cdots$, where $w$ is the local coordinate along the grating, oriented in the direction of the light propagation, and equal to zero at the center of the grating ruled surface. The grating equation gives the $\alpha_{0}$ and $\beta_{0}$ for $E_{0}$. We can then equalize the contribution to the LW coming from the source size and the detector resolution. By also imposing the condition on the total length, we get thus the entrance and exit arms $r_{1}$ and $r_{2}$ at $E_{0}$. By then imposing the condition of focalization and the incidence angle on the detector, we obtain the radius of curvature $R$ and the linear VLS parameter $a_{1}$. Finally we can estimate $a_{2}$ by using one of the expressions found in the literature for the cancellation of the coma aberration. In our case we found that the optimal $a_{2}$ value obtained with the ray tracing is rather different from the one of analytical formulations, probably because in the ray tracing also all the other higher order optical aberrations are intrinsically included, so that $a_{2}$ is used to minimize the contributions from all of them. All the used equations are given in the Appendix.

The optimized parameters are $a_{0}=3200 \mathrm{~mm}^{-1}$, $a_{1}=0.9 \mathrm{~mm}^{-2}, \quad a_{2}=1.2 \times 10^{-4} \mathrm{~mm}^{3}, \quad$ and $\quad a_{3}=a_{4}=0$, $R=58.55 \mathrm{~m}$ (radius measured on the actual substrate before the ruling procedure). The $a_{2}$ parameter is optimized at given $r_{1}$ and $\alpha$ for only one energy, but for a given $a_{2}$ it is possible to cancel the coma aberration by choosing other values of $\alpha$ and $r_{1}$. The set of convenient $\alpha$ and $r_{1}$ is given in Fig. 2 for different photon energies (bottom panel). The horizontal lines indicate the accessible $r_{1}$ range for SAXES: clearly for all the energies it is possible to cancel the coma aberration in more than one way. This explains in a more quantitative way our choice of having precise movements for $\alpha$ and $r_{1}$. The results of Fig. 2 also make clear that the $a_{2}$ parameter is by no means critical and could be set to zero. Nevertheless we adopted the above tiny value with marginal benefits pointed out by extensive ray-tracing work. It must also be noted that Fig. 2 was obtained using the analytical expressions, because the results are very close to those given by the ray tracing (data not shown but obtained for many cases).

This design corresponds to a complete compensation of 


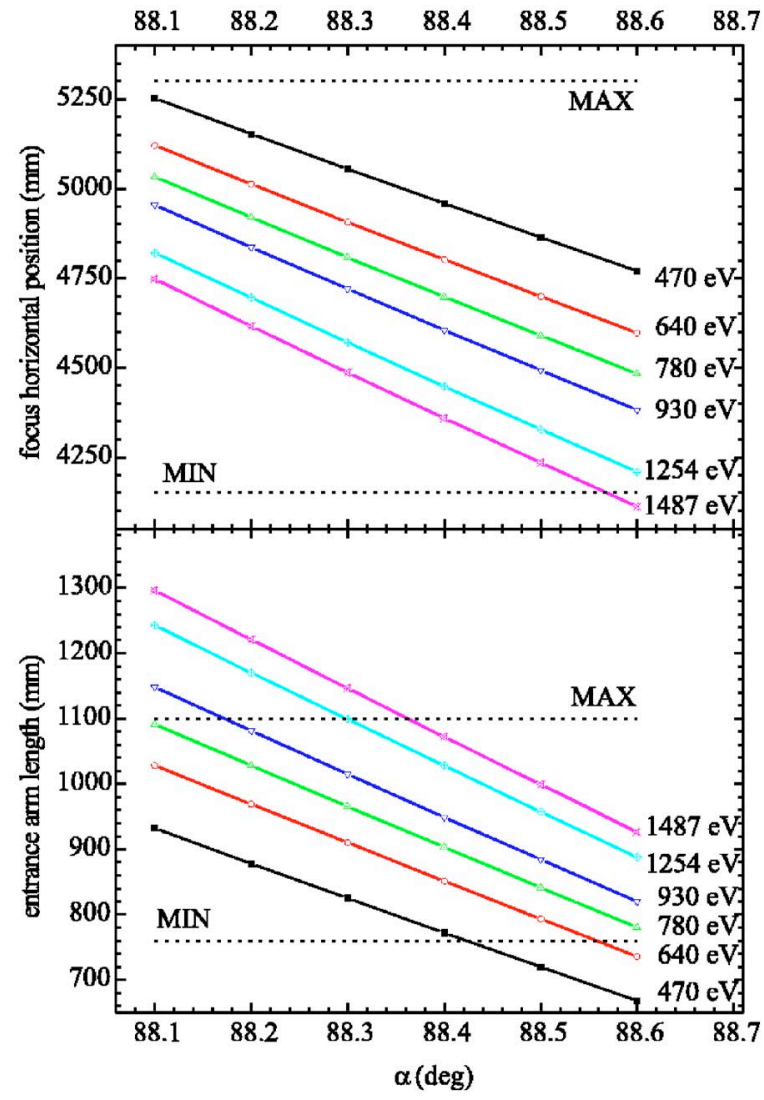

FIG. 2. (Color online) The set of optical parameters minimizing the coma aberration contribution over the whole SAXES energy range. Lower panel: incidence angle on the grating $(\alpha)$ and entrance arm length $r_{1}$. The MIN and MAX lines indicate the limits of $r_{1}$ allowed by the actual SAXES mechanics. Top panel: the corresponding values of the total horizontal length of SAXES. all the aberrations with a vertical opening of $2 \mathrm{mrad}$. In this case the line shape simulated by ray tracing is symmetric within $3 \%$. Moreover, the resolving power is slightly dependent on the working point at the minimum coma and this is a further degree of freedom. In the working conditions of Fig. 2 , the magnification is always around 1 as desired.

\section{THE SAXES COMPONENTS AND THE EXPERIMENTAL SETUP}

An overview of the instrument is given in Fig. 3, where some of the mechanisms holding the vacuum chambers and bellows are not shown for clarity. It must be noted that all the movements and structural parts are independent of the vacuum chambers, in order to maximize the stability of the system. In the drawing we show also the $5 \mathrm{~m}$ long girder on which the spectrometer is installed; this ensures the rigidity necessary to move the spectrometer on the beamline floor (air cushion system) without losing the delicate internal optical alignment. Some technical questions deserve to be briefly discussed. We list them following the path of the light neglecting all the trivial information on conventional techniques (vacuum, slide mechanics, and data reduction).

\section{A. Input slit}

The input slit protrudes into the sample chamber and it is mounted onto a slide mechanism to be possibly placed very close to the sample (minimum of $5 \mathrm{~mm}$ ) in order to optimize the optical matching. The whole slit mechanics has been designed and built in house. It is operated by an accurate $(0.1 \mu \mathrm{m})$ actuator in air through a leverage and a bellow with antagonist springs. The schematic leverage system adopted to ensure a parallel opening of the two blades is

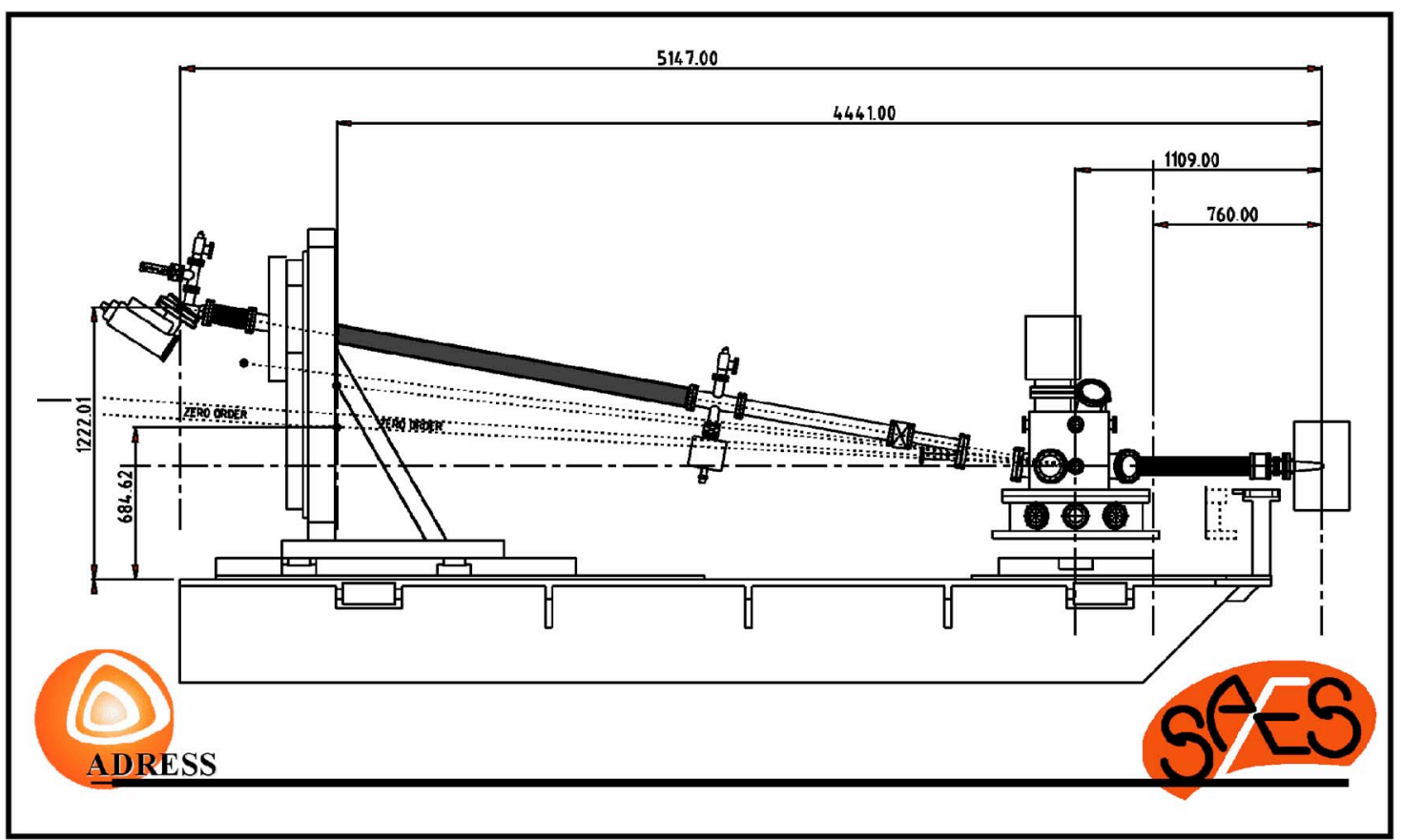

FIG. 3. (Color online) The mechanical simplified construction draft of SAXES. The sample is at the right side. The big grating chamber (600 mm internal diameter) houses the motorized in vacuum mechanics with four degrees of freedom. The whole spectrometer is supported by a single steel girder allowing fine regulation of the vertical position. The girder can rotate around a vertical axis passing on the sample, thanks to an air cushion device; this will allow the study of transferred photon momentum dependent RIXS spectra. 


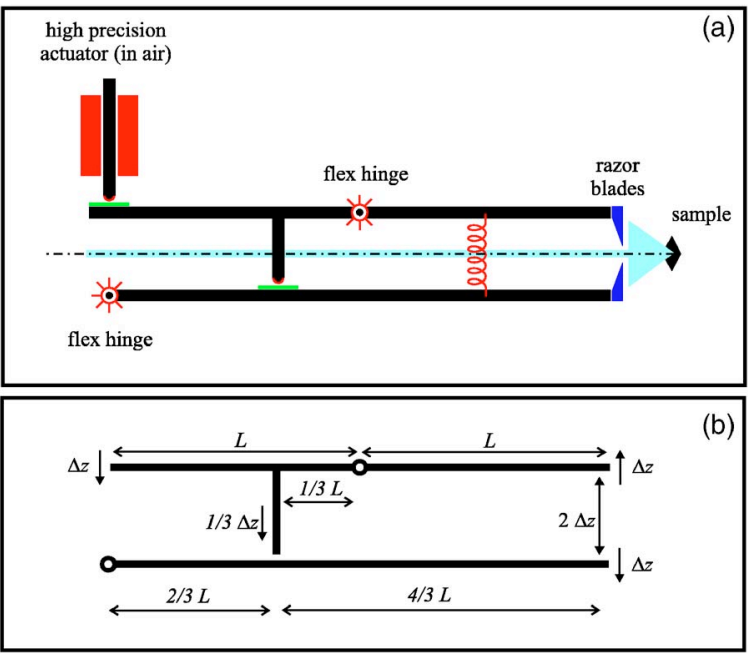

FIG. 4. (Color online) The schematic entrance slit mechanical layout. The two blades open symmetrically, thanks to the choice of the lever arms. One single high precision actuator acts, from the air side, in the leverage. The rotation is provided by two metallic flexible hinges. The long arms are needed to place the actuator along the entrance arm, away from the sample vacuum chamber. Panel (A): The beam proceeds, inside the slit mechanics, from the sample towards the grating after having been selected by the slit. A couple of in vacuum springs are used to counterbalance the actuator. Panel (B): The simple leverage ratios that bring to a 1:2 opening of the slit with respect to the actuator movement $(L=140 \mathrm{~mm})$.

shown in Fig. 4. The slit can be opened to a large value (about $1 \mathrm{~mm}$ ) when slitless operation is desired. In such a case the source for the grating is directly the spot on the sample. The whole slit mechanism is within a metallic protection to prevent stray visible light to enter the spectrometer and to reduce drastically the coupling of the vacuum in the input arm and in the sample chamber.

\section{B. Mask}

A mask, having several apertures of different vertical openings and placed just before the grating, is used to define the vertical aperture, i.e., the illuminated area on the grating surface (see Fig. 1). The vertical movement of the mask is motorized and computer controlled.

\section{Grating}

The grating (substrate in Pyrex, reflective coating in platinum) manufactured by Horiba Jobin Yvon is holographically made by ion etching of the laminar profile grooves. ${ }^{21}$ The holography approximates very well the polynomial density law given above (the theoretical maximum error on the local groove density is $\left.0.006 / 3200=2 \times 10^{-6}\right)$. This high accuracy is possible because the corrections to the constant density are rather modest. The grating dimensions are 20 $\times 130 \times 15 \mathrm{~mm}^{3}$. The efficiency estimated by the manufacturer ranges between $3 \%$ and $4 \%$ over the whole range at $88.3^{\circ}$ incidence angle.

\section{Grating mechanics}

The regulation of the input arm length is obtained by displacing longitudinally the entire vacuum chamber housing the grating with a computer controlled slide mechanism. All

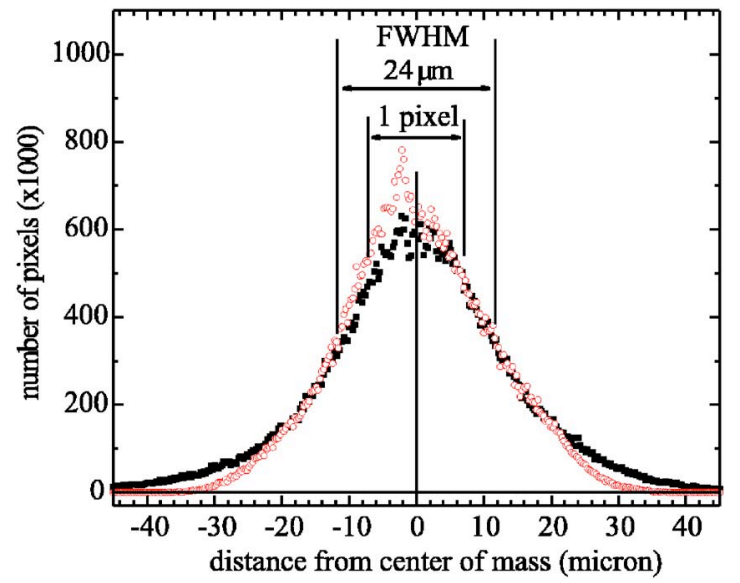

FIG. 5. (Color online) The histogram of the charge cloud distribution on the CCD detector in the event of a single photon detection. The pixel lateral size is $13.5 \mu \mathrm{m}$. In both directions of the CCD array (full black squares perpendicular to energy dispersion direction, open red circles along the dispersion direction), the full width at half maximum of the curve is around $24 \mu \mathrm{m}$, i.e., almost twice the pixel size. See text for details on the procedure used to construct this histogram.

the other movements of the grating (pitch for changing $\alpha$, rolling, vertical positioning, and horizontal transverse movement to allow the use of a second, easily selectable grating) are high precision in vacuum motorized movements manufactured by Micos $\mathrm{GmbH}$.

\section{E. CCD detector}

The CCD detector is a special delivery by Princeton Instruments (Roper Scientific Inc.) ${ }^{22}$ and is based on a thinned and back-illuminated wafer $27.6 \times 27.6 \mathrm{~mm}^{2}$ with $2048 \times 2048$ pixels $\left(13.5 \times 13.5 \mu \mathrm{m}^{2}\right)$. It is mounted on a CF $150 \mathrm{DN}$ flange in such a way to protrude into the vacuum chamber by about $22 \mathrm{~mm}$. In this way the flange can be mounted inclined so that $\gamma$ is about $20^{\circ}$ (see Fig. 1) without shadowing the wafer. The angle $\gamma$ can be adjusted by rotating the camber of the detector from $10^{\circ}$ to $30^{\circ}$. With a mounting of this type it is impossible to use a Peltier cooling to sufficiently reduce the dark current. Thus the detector is cooled with a liquid nitrogen cryostat. The electronically controlled operating temperature is $-100{ }^{\circ} \mathrm{C}$ and the dark current is practically zero ( 1 electron/h pixel), so that the system adds to the statistical fluctuation only the readout noise ( 5.5 electrons/pixel at $100 \mathrm{kHz}$ ).

The spatial resolution of the detector is a crucial parameter and it has been measured in a special experiment whose results are summarized in Fig. 5. We have illuminated the detector with a uniform and weak flux of $\mathrm{x}$ rays from a traditional source $(\mathrm{Cu}$ anode, $930 \mathrm{eV}$ energy) for a short time (around $1 \mathrm{~s}$ ) at a distance of $2 \mathrm{~m}$. The low exposure ensures that the vast majority of the photons appears in the image as isolated spots over the dark background (no double events). Due to the photon absorption and charge release mechanisms in the CCD wafer, a single photon results in a charge cloud shared by a number of neighboring pixels (split events), and only seldom the whole charge is in a single pixel. This fact implies that the effective spatial resolution of the CCD is worse than the pixel size, and it has been shown that it is 


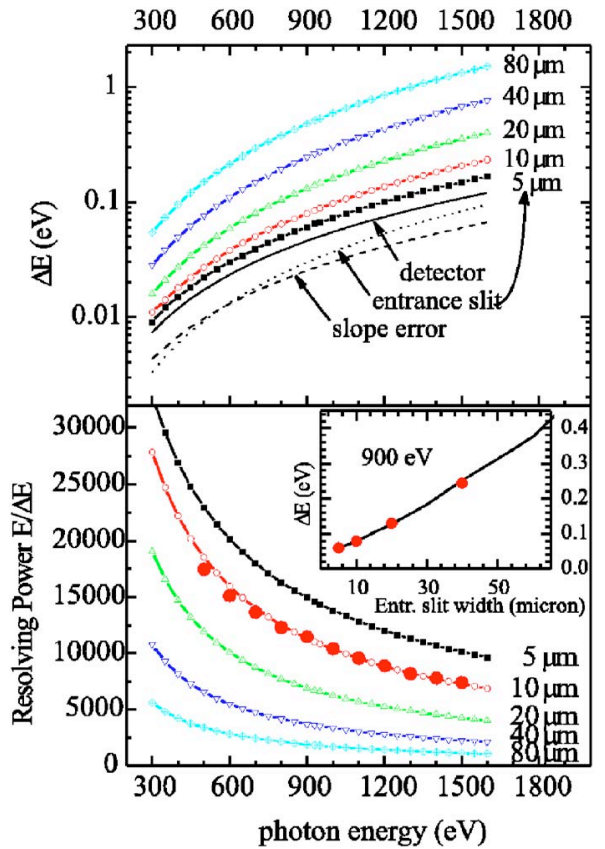

FIG. 6. (Color online) The expected resolution performances of SAXES. Top panel: The expected linewidth (LW) vs photon energy for various openings of the entrance slit (small symbols). For the minimum slit $(5 \mu \mathrm{m})$, the slit contributions compared to the expected contributions from the grating slope error and CCD spatial resolution (with $\gamma=20^{\circ}$ ). Bottom panel: The corresponding values of the resolving power $E / \Delta E$. The big red circles indicate the resolving power estimated by ray tracing; all the other values are estimated by analytical expressions. In the inset the LW dependence on the slit opening at the important case of $900 \mathrm{eV}$.

almost independent of it. ${ }^{23}$ We have statistically analyzed this phenomenon over thousands of those spots; the results are shown in Fig. 5, where the histogram of the charge spatial distribution is given for the two dimensions of the detector. For each spot we have determined the center of mass of the charge accumulated in the various pixels, along rows and columns, with an accuracy of $1 \mu \mathrm{m}$ in the CCD space; then we have attributed the weight of each pixel of that spot to the corresponding distance from the center of mass; and repeating this procedure for thousands of spots results in the smooth and highly resolved histogram of Fig. 5. In the specific case of our CCD, the FWHM of the histogram is about $24 \mu \mathrm{m}$. It is important to remark that the average charge cloud is the same in the two directions, despite the grazing incidence. This fact further supports the use of CCD detectors at grazing incidence.

\section{THE PERFORMANCES OF SAXES}

The typical theoretical average resolving power with various openings of the input slit over the whole working energy range is given in Fig. 6 showing values fully consistent with the general goals of the project. In this evaluation the coma contribution is neglected in the analytical evaluation of the LW (small symbols in both panels), whereas the requested slope error $(0.97 \mu \mathrm{rad})$ is taken into account. From the top panel it appears clearly that the slope error contribution is negligible already with $S_{1}=5 \mu \mathrm{m}$. The measured slope error is $0.67 \mu \mathrm{rad}$, better than the requested $0.97 \mu \mathrm{rad}$, so in the ray tracing we neglected the grating slope error. The

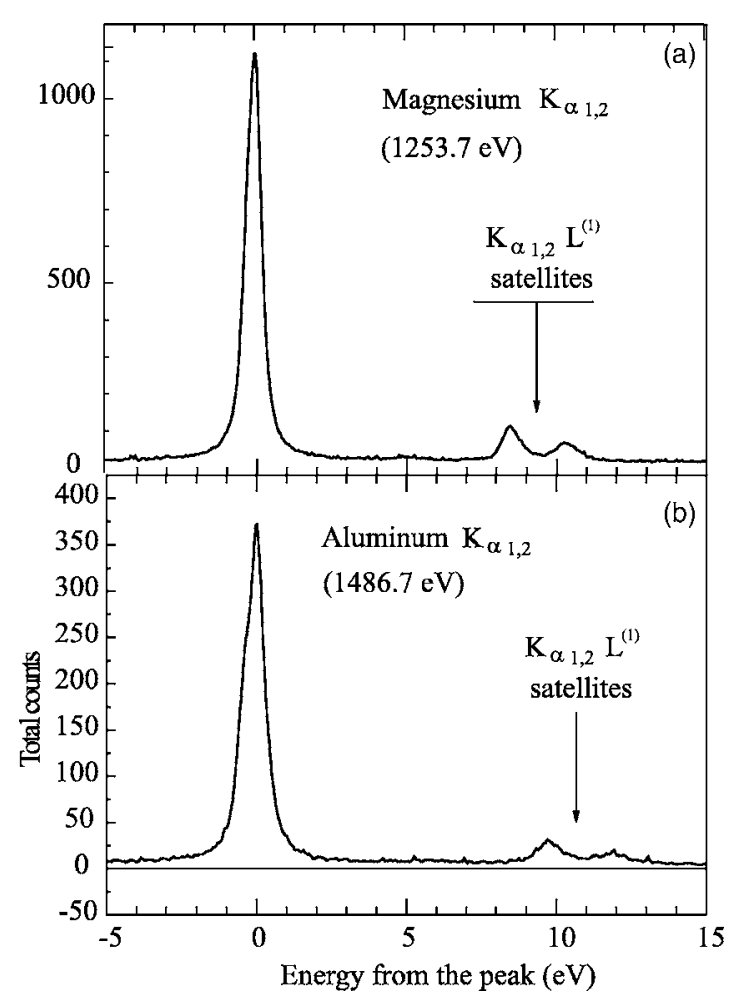

FIG. 7. The $\mathrm{Mg}$ and $\mathrm{Al} K_{\alpha 1,2}$ emission spectra measured with SAXES. The $K_{\alpha 1,2} L^{1}$ satellites are used to calibrate in a precise way the energy scale by comparison with published spectra. The calibration is in good agreement with the expected one. The main peak in both cases is asymmetric due to the $2 p$ level spin-orbit splitting. The spectra were measured with a $10 \mu \mathrm{m}$ entrance slit and an accumulation time of about $20 \mathrm{~min}$. See text for details.

ray-tracing results for $S_{1}=10 \mu \mathrm{m}$ are shown in the bottom panel by the big circles; the agreement with the analytical results is excellent. In consideration of the expected spot size on the sample $(9 \mu \mathrm{m})$, we consider as ultimate performances of SAXES those obtained with $S_{1}=10 \mu \mathrm{m}$, also because the main contribution to the LW comes already from the CCD spatial resolution.

The SAXES performances have been tested with the fluorescence emission lines $\mathrm{Al} K_{\alpha 1,2}$ and $\mathrm{Mg} K_{\alpha 1,2}$ that are the only easily accessible options with a conventional $\mathrm{x}$-ray tube. This has the advantage of providing a test independent from the performances of the beamline where the instrument will be installed. Another advantage is that these lines (1486.7 and $1253.7 \mathrm{eV}$ ) are at the upper energy side of the SAXES range so that the test is very severe and really significant. Due to the spin-orbit splittings of the $2 p$ shallow levels, the $K_{\alpha}$ emissions of $\mathrm{Al}$ and $\mathrm{Mg}$ are the superposition of two transitions to the $K$-shell hole from the $2 p_{1 / 2}$ and $2 p_{3 / 2}$ levels, respectively, giving two contributions with intensities in the ratio of 1:2. The parameters (i.e., spin orbit and lifetime broadening) are known from the literature and, in particular, from the NIST benchmark work, ${ }^{24}$ where the two emission spectra were measured with a crystal spectrometer, much more effective at these energies both in resolving power and angular acceptance.

The raw data measured with $10 \mu \mathrm{m}$ input slit and a mask reducing the vertical acceptance to $1 \mathrm{mrad}$ are given in Fig. 7 in a $20 \mathrm{eV}$ wide energy interval, i.e., from $5 \mathrm{eV}$ below the peak to $15 \mathrm{eV}$ above the peak. In this energy range also 


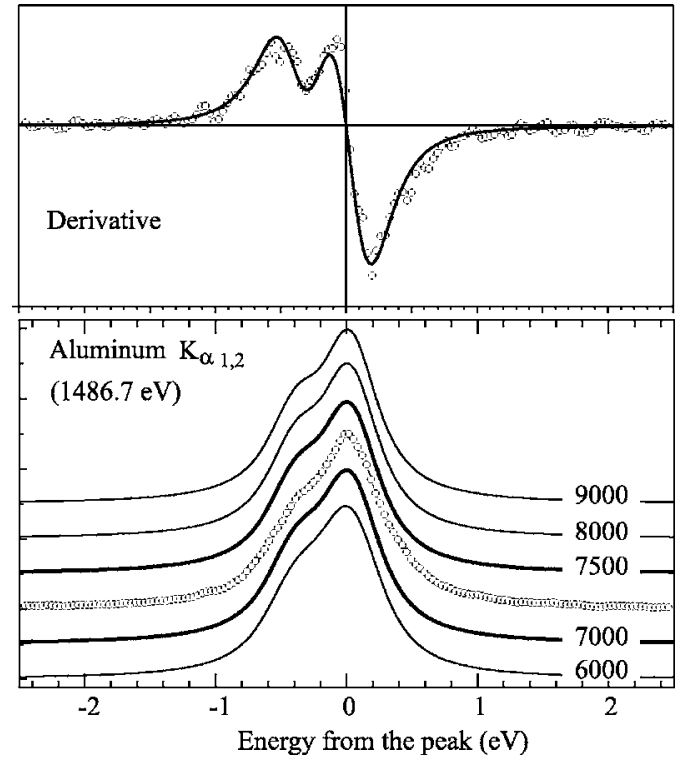

FIG. 8. To determine the actual resolving power of SAXES in the case of the $\mathrm{Al}$ spectrum of Fig. 6, we compare the experimental data to the simulated spectra obtained by a Gaussian broadening of the ideal spectrum measured with infinite resolving power. It must be noted that the Lorentzian lifetime broadening is unavoidable in the fluorescence emission lines. In the top panel the comparison is made using the derivatives of the measured and simulated spectra. At $1486.7 \mathrm{eV}$ SAXES can clearly work with a resolving power better than 7000 .

the $K \alpha L$ satellites due to fluorescence emission in the presence of multiple ionization are clearly seen. Since the satellite energy is known from the literature, ${ }^{25}$ it is used to check the calibration of the spectra. A good point is that only a very weak scattered radiation background is present in the measured spectra, and this is particularly important in consideration of the bremsstrahlung contribution from the electron excited source (electron energy around 5-6 keV). Already in this scale of the drawing, the Lorentzian tails of the peaks due to finite lifetime are apparent. Moreover, it is seen that the $\mathrm{Al}$ line is clearly not a single peak. This is very crucial in the analysis of the resolution as shown in Fig. 8 where the peak is presented in an expanded scale (open points in lower panel). The experimental spectrum is compared with the theoretical spectra expected with various resolving powers. It is seen at a glance that the resolving power is greater than 6000 since the shoulder from the $2 p_{1 / 2}$ transition is better seen in the experiment. On the other hand the resolving power is lower than 9000. Within the accuracy of the analysis, the measured spectrum is rather well compared with the spectra having 7000 and 7500 resolving powers given by heavy solid lines sandwiching the experimental spectrum. One can thus conclude that the resolving power is typically between 7000 and 7500 at $1486.7 \mathrm{eV}$, i.e., at the upper side of the SAXES working range in excellent agreement with the expectations summarized in Fig. 6. This evaluation has rather small uncertainty since the parameters used in the simulated spectra are rather well known from Ref. 24; the spin-orbit energy splitting is $0.413 \mathrm{eV}$ with an accuracy of $\pm 1 \mathrm{meV}$ and it does not introduce ambiguity. The Lorentzian parameter $\Gamma$ $=0.45 \mathrm{eV}(\mathrm{FWHM})$ is in the numerical range given in the NIST paper ${ }^{24}$ and is obtained by fitting the tails of the mea- sured spectrum in perfect agreement with our fitting of the NIST spectrum itself. In fact, the lifetime parameter determines completely the tails when the resolving power is above about $5000-6000$ so that $\Gamma$ is obtained safely from the data without making any assumption on the resolution. A further confirmation of the above resolution analysis is given by the derivative of the spectra having the advantage of enhancing the fingerprints of the doublet nature of the peak. This is given in the upper panel of Fig. 8 where the open dots are the experiment and the solid line is the theoretical spectrum expected with a resolving power of 7250 . The general agreement is excellent. The transition from positive to negative derivative across the peak is perhaps sharper in the experiment, suggesting a resolving power slightly above the value estimated above. However, the noise makes this refinement of the analysis rather difficult. This is not done because it is beyond the scope of the present work.

A similar analysis has been done also with the $\mathrm{Mg}$ spectrum. As it is apparent from Fig. 8 and from Ref. 24 the $\mathrm{Mg}$ peak is narrower than in Al. This is due to a smaller spin orbit $(0.251 \mathrm{eV})$ and to the narrower instrumental linewidth expected at this energy that is $233 \mathrm{eV}$ lower. However, the analysis of the type done in $\mathrm{Al}$ has greater uncertainties in $\mathrm{Mg}$ because the Lorentzian broadening masks substantially the shoulder due to the smaller spin-orbit splitting as it is apparent also from the benchmark case. The result is a resolving power between 8000 and 10000 which is in any case a further support to the good performances of SAXES. In fact, this value is consistent with the theoretical trend given in Fig. 6, confirming the successful performance of the instrument presented here.

Based on these tests we can safely predict that SAXES will be able to reach the targeted resolving power (higher than 10000 ) for the whole $400-1100 \mathrm{eV}$ range. The extreme resolution performances, the optimized optical matching to the dedicated ADRESS beamline, and the possibility of changing the scattering angle make SAXES a rather unique tool for future RIXS spectroscopy on strongly correlated electron systems.

\section{ACKNOWLEDGMENTS}

The authors are indebted to F. van der Veen for encouragement and precious supervision of the project. The authors thank N. B. Brookes for his wise scientific and technical suggestions and F. Romanato for the willing support in the search for high quality blades for the SAXES entrance slit. They also acknowledge the excellent technical support provided by C. Farioli and A. Moroni. SAXES is a joint project of the Paul Scherrer Institut (PSI) and of the Politecnico di Milano. The SAXES spectrometer is property of the Swiss Light Source at the PSI.

\section{APPENDIX}

We summarize here the procedure we followed to determine the main optical parameters of SAXES. The used equations are taken from Refs. 16 and 18. The list of symbols is the following: $\alpha=$ incidence angle on the grating, 


$$
\begin{aligned}
& \beta=\text { diffracted beam angle, positive } \\
& a(w)=a_{0}+a_{1} w \\
& +a_{2} w^{2}+a_{3} w^{3}+\cdots=\text { local groove density on the grating } \\
& \text { (in } \mathrm{mm}^{-1} \text { ), } \\
& r_{1}=\text { entrance arm, } \\
& r_{2}=\text { exit arm, } \\
& R=\text { radius of curvature of the grating } \\
& \text { surface, } \\
& E_{0}=\text { reference photon energy, } \\
& L=r_{1}+r_{2,0}=\text { total length at } E_{0} \text {, } \\
& S_{1}=\text { source size (in the dispersion } \\
& \text { direction), } \\
& S_{2}=\text { CCD spatial resolution, } \\
& \gamma=\text { grazing incidence angle on the } \\
& \theta=(\alpha+\beta) / 2 \text {, } \\
& k=+1=\text { diffraction order (first internal), } \\
& K=1.239842 \\
& \times 10^{-3} \mathrm{~mm} \mathrm{eV}=\text { energy to wavelength conversion, } \\
& s^{\prime}=0.2 \operatorname{arc~sec} \\
& =0.96 \mu \mathrm{rad}=\text { grating surface slope error. }
\end{aligned}
$$

Some of the parameter values are predetermined by external requirements or technological limitations $\left(S_{1}=8 \mu \mathrm{m}, S_{2}\right.$ $=28 \mu \mathrm{m}, a_{0}=3200 \mathrm{~mm}^{-1}$, and $\left.L=5 \mathrm{~m}\right)$ and others are chosen for scientific reasons (energy range of $400-1600 \mathrm{eV}$, $E_{0}=900 \mathrm{eV}$ ). Thus, given $E_{0}, \theta_{0}, a_{0}$, and $k$, from the grating equation we get

$$
\varphi_{0}=\frac{\alpha-\beta}{2}=\arcsin \frac{a_{0} k K}{2 \cos \theta_{0} E_{0}},
$$

$$
\begin{aligned}
& \alpha=\theta_{0}-\varphi_{0}, \\
& \beta_{0}=\theta_{0}+\varphi_{0} .
\end{aligned}
$$

Considering $\left(S_{2} \sin \gamma\right)$ the effective spatial resolution of the detector at grazing incidence, the contributions to LW coming from $S_{1}$ and $S_{2}$ are

$$
\begin{aligned}
& \Delta E_{1}=S_{1} \frac{\cos \alpha}{r_{1} a_{0} k K} E^{2}, \\
& \Delta E_{2}=S_{2} \sin \gamma \frac{\cos \beta}{r_{2} a_{0} k K} E^{2},
\end{aligned}
$$

By requiring $\Delta E_{1}=\Delta E_{2}$ and $r_{1}+r_{2}=L$, we find

$$
\begin{aligned}
& r_{2,0}=\frac{S_{2} \cos \beta_{0}}{S_{1} \cos \alpha+S_{2} \cos \beta_{0}} L, \\
& r_{1}=L-r_{2,0} .
\end{aligned}
$$

We can now impose that the system be on focus,

$$
\frac{\cos ^{2} \alpha}{r_{1}}+\frac{\cos ^{2} \beta_{0}}{r_{2,0}}=\frac{\cos \alpha+\cos \beta_{0}}{R}+\frac{k K}{E_{0}} a_{1},
$$

and that the focal curve be tangent to the CCD detector,

$$
\frac{1}{r_{2}} \frac{\cos \beta_{0}}{\tan \gamma}+\frac{\tan \beta_{0}}{R}+\frac{a_{1}}{a_{0}}=\frac{2 \sin \beta_{0}}{r_{2,0}},
$$

and we can obtain $R$ and $a_{1}$ :

$$
\begin{aligned}
& R=\frac{\left(E_{0} / k K\right)\left(\cos \alpha+\cos \beta_{0}\right)-a_{0} \tan \beta_{0}}{\left(E_{0} / k K\right)\left[\left(\cos ^{2} \alpha\right) / r_{1}+\left(\cos ^{2} \beta_{0}\right) / r_{2}\right]-\left(a_{0} / r_{2,0}\right)\left[2 \sin \beta_{0}-\left(\cos \beta_{0}\right) /(\tan \gamma)\right]}, \\
& a_{1}=\frac{E_{0}}{k K}\left(\frac{\cos ^{2} \alpha}{r_{1}}+\frac{\cos ^{2} \beta_{0}}{r_{2,0}}-\frac{\cos \alpha+\cos \beta_{0}}{R}\right) .
\end{aligned}
$$

Finally we find $a_{2}$ with the following equation, obtained by modifying the ones given in Ref. 16 or in Ref. 18 (which, incidentally, do not coincide) and "optimized" empirically by comparing the results with ray tracing:

$$
\begin{aligned}
a_{2}= & \frac{3}{8} \frac{E_{0}}{k K}\left[\left(\frac{1}{R}-\frac{\cos \alpha}{r_{1}}\right) \frac{\sin 2 \alpha}{r_{1}}\right. \\
& \left.-\left(\frac{1}{R}-\frac{\cos \beta_{0}}{r_{2,0}}\right) \frac{\sin 2 \beta_{0}}{r_{2,0}}\right] .
\end{aligned}
$$

For $E_{0}$ we know now $r_{1}, r_{2}, \alpha$, and $\beta_{0}$ and we have assigned the grating parameters $R, a_{0}, a_{1}$, and $a_{2}$. For any $E$ we can thus calculate $\beta, r_{2}$, and $\gamma$, having decided in advance what $\alpha$ and $r_{1}$ one wants to use (see Fig. 2),

$$
\beta=\arcsin \left(\sin \alpha-\frac{k K}{E} a_{0}\right),
$$

$$
\begin{aligned}
& r_{2}=\frac{\cos ^{2} \beta}{(\cos \alpha+\cos \beta) / R-\left(\cos ^{2} \alpha\right) / r_{1}+(k K / E) a_{1}}, \\
& \gamma=\arctan \frac{\cos \beta}{2 \sin \beta-r_{2}\left[(\tan \beta) / R+a_{1} / a_{0}\right]} .
\end{aligned}
$$

The contributions to the $\mathrm{LW}$ from the finite size of the source, the spatial resolution of the CCD, and the grating surface slope error are then

$$
\Delta E_{1}=S_{1} \frac{\cos \alpha}{r_{1} a_{0} k K} E^{2},
$$

$$
\Delta E_{2}=S_{2} \sin \gamma_{r} \frac{\cos \beta}{r_{2} a_{0} k K} E^{2},
$$




$$
\Delta E_{\mathrm{SE}}=\frac{2 s^{\prime}}{\tan (\alpha-\beta)} E .
$$

It is very difficult to give a reliable estimate of the $\Delta E$ coming from the coma aberration, because the analytical expressions given in the literature are not unanimous and because the effects of coma are not symmetric on the energy peak and are quadratic with the footprint length on the grating. We limit ourselves to consider it small because we know that for every energy it is possible to minimize it more or less empirically. In the calculations of LW and resolving power, we have quadratically summed the three contributions. As already mentioned in Sec. II all the parameters were checked by ray tracing and the best value of $a_{2}$ was also found by simulations; it must be considered that other higher order aberrations can give a contribution, so the optimized value of $a_{2}$ can be different from the calculated one because it is utilized to reduce also types of aberrations other than coma.

${ }^{1}$ A. Kotani and S. Shin, Rev. Mod. Phys. 73, 203 (2001).

${ }^{2}$ J. Nordgren, J. Electron Spectrosc. Relat. Phenom. 110-111, ix (2000).

${ }^{3}$ A. Kotani, Eur. Phys. J. B 47, 3 (2005).

${ }^{4}$ T. A. Callcott, K. L. Tsang, C. H. Zhang, D. L. Ederer, and E. T. Arakawa, Rev. Sci. Instrum. 57, 2680 (1986).

${ }^{5}$ J. Nordgren, J. Bray, S. Gramm, R. Nyholm, J.-E. Rubensson, and N. Wassdahl, Rev. Sci. Instrum. 60, 1690 (1989).

${ }^{6}$ S. Shin, A. Agui, M. Fujisawa, Y. Tezuka, and T. Ishii, Rev. Sci. Instrum. 66, 1584 (1995).

${ }^{7}$ C. Dallera, E. Puppin, A. Fasana, G. Trezzi, N. Incorvaia, L. Braicovich,
N. B. Brookes, and J. B. Goedkoop, J. Synchrotron Radiat. 3, 231 (1996).

${ }^{8}$ D. Cocco, M. Matteucci, K. C. Prince, and M. Zangrando, Proc. SPIE 4506, 46 (2001); D. Cocco et al., AIP Conf. Proc. 705, 873 (2004).

${ }^{9}$ C. F. Hague, J. H. Underwood, A. Avila, R. Delaunay, H. Ringuenet, M. Marsi, and M. Sacchi, Rev. Sci. Instrum. 76, 023110 (2005).

${ }^{10}$ Y. Chuang, J. Pepper, W. McKinney, Z. Hussain, E. Gullikson, P. Batson, D. Qian, and M. Z. Hasan, J. Phys. Chem. Solids 66, 2173 (2006).

${ }^{11}$ T. Tokushima, Y. Harada, H. Ohashi, Y. Senba, and S. Shin, Rev. Sci. Instrum. 77, 063107 (2006).

${ }^{12}$ G. Ghiringhelli, A. Tagliaferri, L. Braicovich, and N. B. Brookes, Rev. Sci. Instrum. 69, 1610 (1998).

${ }^{13}$ J. Chavanne, P. Van Vaerenbergh, P. Elleaume, and T. Gunzel, Proceedings of EPAC 2000, Vienna, Austria, 2000, p. 2346.

${ }^{14}$ G. Ghiringhelli et al., Phys. Rev. Lett. 92, 117406 (2004).

${ }^{15}$ J.-M. Mariot, M. Sacchi, L. Journel, J.-J. Gallet, M. McElfresh, and C. F. Hague, Nucl. Instrum. Methods Phys. Res. B 246, 176 (2006).

${ }^{16}$ K. D. Osborn and T. A. Callcott, Rev. Sci. Instrum. 66, 3131 (1995).

${ }^{17}$ W. R. McKinney, Rev. Sci. Instrum. 63, 1410 (1992).

${ }^{18}$ M. R. Howells, in X-ray Data Booklet, edited by A. Thompson et al. (Lawrence Berkeley National Laboratory, Berkeley, 2001), http:// xdb.lbl.gov/

${ }^{19}$ The original SHADOW package is available at http:// www.nanotech.wisc.edu/CNTLABS/shadow.html and the graphical user interfaces for personal computer at http://www.esrf.fr/computing/ scientific/xop/

${ }^{20}$ H. Noda, T. Tamioka, and N. Seya, J. Opt. Soc. Am. 64, 1031 (1974).

${ }^{21} \mathrm{http} / / / \mathrm{www}$.jobinyvon.com/usadivisions/VUV/variable line.htm

${ }^{22}$ http://www.piacton.com/products/pisx/

${ }^{23}$ E. Dinardo et al., Nuc. Instrum. Methods Phys. Res. A (in press).

${ }^{24}$ J. Schweppe, R. D. Delattes, T. Mooney, and C. J. Powell, J. Electron Spectrosc. Relat. Phenom. 67, 463 (1994).

${ }^{25}$ O. Mauron, J.-Cl. Dousse, J. Hoszowska, J. P. Marques, F. Parente, and M. Polasik, Phys. Rev. A 62, 062508 (2000). 